\title{
Generalized rough sets based on reflexive relations*
}

\author{
Yu-Ru SyAu AND LIXING JiA ${ }^{\dagger}$
}

\begin{abstract}
We study the approximation theory of a special kind of neighborhood systems, called total pure reflexive neighborhood systems, which is a generalization of pretopological and topological neighborhood systems. In the framework of such neighborhood systems, the so-called lower and upper approximations are considered. For a pretopological neighborhood system on a fixed nonempty set $U$, we show that the family $\mathcal{T}$ of fixed points of its lower approximation is a topology for $U$, and establish a characterization (in terms of neighborhoods) of $\mathcal{T}$-open sets.

We then regard a reflexive relation $R$ on $U$ as the total pure reflexive neighborhood system $x \longmapsto\{R(x)=\{y \in U \mid(x, y) \in$ $R\}$, whose induced upper approximation is identical to the commonly used upper approximation $R^{*}: 2^{U} \rightarrow 2^{U}$ based on $R$. We show that the family $\mathcal{T}_{R}$ of all subsets $X$ of $U$ for which $R^{*}(U-X)=U-X$ is an Alexandroff topology for $U$, and that the pre-topologically maximal neighborhood system of the neighborhood system $x \longmapsto\{R(x)\}$ associated to $R$ is exactly the pretopological neighborhood system whose induced upper approximation is identical to $R^{*}: 2^{U} \rightarrow 2^{U}$. Accordingly, we show that for each $x \in U$, its smallest $\mathcal{T}_{R}$-open neighborhood is the intersection of all $\mathcal{T}_{R}$-open sets containing $R(x)$. In addition, we study the so-called $R$-definability. We establish a characterization of $R$-definable sets in terms of $R^{*}$ and its dual $R_{*}$, and present a necessary condition for $R$-definability.
\end{abstract}

KEYWORDS AND PHRASES: Rough sets, reflexive relations, neighborhood systems, preclosures, pretopological neighborhood systems.

\section{Introduction}

The fundamental concepts of rough set theory are the lower and upper approximations $[12,13]$ defined in a Pawlak's approximation space. Namely, an ordered pair $(U, \beta)$ consists of a fixed nonempty set $U$ of all the objects

\footnotetext{
${ }^{*}$ Supported by the National Science Council of TAIWAN under contract NSC 101-2221-E-150-056.

${ }^{\dagger}$ Please address all correspondences to Lixing Jia. E-mail: ljia@csu.edu.
} 
under consideration and an indiscernibility relation $\beta \subseteq U \times U$ which is assumed to be an equivalence relation.

Some generalizations of Pawlak's approximation spaces have been made by considering weaker forms of an indiscernibility relation instead of an equivalence relation $[4,8,15]$. We will simply call such generalizations approximation spaces.

Slowinski and Vanderpooten [15] suggested that the reflexivity property seems quite necessary to express any form of indiscernibility or similarity, and proposed new definitions of lower and upper approximations, $R_{*}(X)$ and $R^{*}(X)$, based on a reflexive relation $R$. As it is known [6], the family of fixed points of such a lower approximation is a topology. However, unlike the lower approximation based on a reflexive and transitive relation [19], the lower approximation based on a reflexive relation is not in general idempotent.

Pawlak's lower and upper approximations are indeed a pair of interior and closure operators on the set $U[12,13]$ : in other words, they are dual and the upper approximation satisfies the Kuratowski closure axioms which define a topological structure on $U$. There are various equivalent ways of defining this structure. This leads us to study the topological structure from different viewpoints.

In this paper, we study the approximation theory of a special kind of neighborhood systems, called total pure reflexive neighborhood systems. In the framework of such neighborhood systems, the so-called lower and upper approximations are considered. For a pretopological neighborhood system on the set $U$, we show that the family $\mathcal{T}$ of fixed points of its lower approximation is a topology for $U$, and establish a characterization (in terms of neighborhoods) of $\mathcal{T}$-open sets.

We then regard a reflexive relation $R$ on $U$ as the total pure reflexive neighborhood system $x \longmapsto\{R(x)=\{y \in U \mid(x, y) \in R\}\}$, whose induced upper approximation is identical to $R^{*}: 2^{U} \rightarrow 2^{U}$. We show that the family $\mathcal{T}_{R}$ of all subsets $X$ of $U$ for which $R^{*}(U-X)=U-X$ is an Alexandroff topology. Namely, a topology in which arbitrary intersections of open sets are open, or equivalently, every point has a minimal open neighborhood [1]. We show that the smallest $\mathcal{T}_{R}$-open neighborhood of each object $x \in U$ is the intersection of all $\mathcal{T}_{R}$-open sets containing $R(x)$. In addition, we study the notion of $R$-definability introduced in [15]. We establish a characterization of $R$-definable sets in terms of $R^{*}$ and its dual $R_{*}$, and present a necessary condition for $R$-definability.

In what follows, we provide some preliminary backgrounds in section 2 . We then present total pure reflexive (TPR) neighborhood systems and pretopological neighborhood systems in section 3 and 4 respectively. In section 5, we consider TPR neighborhood systems under a reflexive relation 
$R$ and characterize $R$-definable sets. We conclude with some remarks and future projects in section 6 .

\section{Preliminaries}

Let $U$ be a certain nonempty set referred as the universe (of discourse). The power set of $U$, denoted by $2^{U}$, is the collection of all subsets of $U$. That is, $2^{U}=\{S \mid S \subseteq U\}$.

We use the symbols $\subseteq$ and $\subset$ to indicate "subset" and "proper subset" respectively.

Let $R \subseteq U \times U$ be a (binary) relation on $U$. We often write $x R y$ instead of $(x, y) \in R$. The inverse relation of $R$, denoted by $R^{-1}$, is obtained by reversing the ordered pairs of $R$. That is, $R^{-1}=\{(x, y) \mid(y, x) \in R\}$.

For $x \in U$, the subset of $U$ defined by $R(x)=\{y \in U \mid x R y\}$ is called the image of $x$ under $R$. Observe that

$$
R^{-1}(x)=\left\{y \in U \mid x R^{-1} y\right\}=\{y \in U \mid y R x\} .
$$

Notice that if $R$ is symmetric then $R(x)=R^{-1}(x)$ for each $x \in U$. If $R$ is an equivalence relation on $U$, then for each $x \in U$ the set $R(x)=R^{-1}(x)$ coincides with the $R$-equivalence class $[x]_{R}$ of $x$, and we designate by $X / R$ the family of all distinct $R$-equivalence classes.

Lemma 1 ([15]). Let $R \subseteq U \times U$ be reflexive. For any $X \subseteq U$, we have

$$
\bigcup_{x \in X} R^{-1}(x)=\{x \in U \mid R(x) \cap X \neq \emptyset\} .
$$

Definition 1 ([15]). Considering a subset $X \subseteq U$ and a binary relation $R$ defined on $U, X$ is $R$-definable if and only if:

$$
\begin{gathered}
\exists X^{\prime} \subseteq X \text { such that } X=\bigcup_{x \in X^{\prime}} R^{-1}(x) \\
\forall x \in X^{\prime}, \quad R(x) \subseteq X .
\end{gathered}
$$

Such a set $X^{\prime}$ is referred to as a reference set of the $R$-definable set $X$.

\subsection{Pawlak's lower and upper approximations}

Let $\beta$ be an equivalence relation on $U$. For any $X \subseteq U$, according to Pawlak [13], a pair of lower and upper approximations, $\beta_{*}(X)$ and $\beta^{*}(X)$, respectively, are defined as follows:

$$
\beta_{*}(X)=\cup\{Y \in U / \beta \mid Y \subseteq X\}, \quad \beta^{*}(X)=\cup\{Y \in U / \beta \mid Y \cap X \neq \emptyset\} .
$$


Equivalently, $\beta(X)$ and $\bar{\beta}(X)$ can be also presented as below:

$$
\beta_{*}(X)=\left\{x \in U \mid[x]_{\beta} \subseteq X\right\}, \quad \beta^{*}(X)=\left\{x \in U \mid[x]_{\beta} \cap X \neq \emptyset\right\} .
$$

\subsection{The notion of topological spaces}

A family $\mathcal{T}$ of subsets of $U$ is called a topology on $U$ if it contains the whole set $U$ and the empty set $\emptyset$, and is closed under finite intersections and arbitrary unions. The pair $(U, \mathcal{T})$ is called a topological space. The members of $\mathcal{T}$ are called $\mathcal{T}$-open, or open sets.

The complement of an open set is called a closed set. Using de Morgan's laws, topology can be alternatively described in terms of closed sets as well; more precisely, $U$ and $\emptyset$ are closed, arbitrary intersections of closed sets are closed, and finite unions of closed sets are closed.

There are many other ways to set up axioms, including the so-called $\mathrm{Ku}-$ ratowski closure axioms (in short, KC-axioms) and the topological neighborhood axioms (in short, TN-axioms), that can be used to define this structure.

2.2.1. Kuratowski closure axioms An operator $c: 2^{U} \longrightarrow 2^{U}$ is called a (topological) closure on $U$ if it satisfies the following four statements (known as the Kuratowski closure axioms):

(KC 1) $c(\emptyset)=\emptyset$ (Preservation of nullary unions).

(KC 2) For each $X, X \subseteq c(X)$ (Extensivity).

(KC 3) For each $X, c(c(X))=c(X)$ (Idempotence).

(KC 4) For each $X$ and $Y, c(X \cup Y)=c(X) \cup c(Y)$ (Additivity).

The last axiom implies the following:

(KC 5) If $X \subseteq Y$ then $c(X) \subseteq c(Y)$ (Order-preserving).

The dual of a closure $c: 2^{U} \longrightarrow 2^{U}$ is the interior $i: 2^{U} \longrightarrow 2^{U}$ defined by

$$
i(X)=U-c(U-X), \quad \forall X \subseteq U .
$$

Then the interior satisfies the so-called Kuratowski interior axioms which are dual to the Kuratowski closure axioms:

(KI 1) $i(U)=U$.

(KI 2) For each $X, i(X) \subseteq X$.

(KI 3) For each $X, i(i(X))=i(X)$ (Idempotence).

(KI 4) For each $X$ and $Y, i(X \cap Y)=i(X) \cap i(Y)$.

The last axiom implies the following:

(KI 5) If $X \subseteq Y$ then $i(X) \subseteq i(Y)$ (order-preserving). 
2.2.2. Topological neighborhood axioms By a neighborhood system on $U$ we mean a mapping $N S: U \longrightarrow 2^{2^{U}}$ which assigns to each $x \in U$ a nonempty collection $N S(x)$ of subsets of $U$ called neighborhoods of $x$.

Note that the broader definition given in $[3,10]$ for a neighborhood system on $U$ doesn't require that $N S(x) \neq \emptyset$ for each $x \in U$.

A neighborhood system $N S: U \rightarrow 2^{2^{U}}$ is called a topological neighborhood system, or a topology, on $U$ if for each $x \in U, N S(x)$ satisfies the following TN-axioms [5]:

(TN 1) If $N \in N S(x)$, then $x \in N$.

(TN 2) If $N, M$ are members of $N S(x)$, then $N \cap M \in N S(x)$.

(TN 3) Superset condition: If $M \supseteq N$ for a nonempty $N \in N S(x)$, then $M \in N S(x)$.

(TN 4) If $N \in N S(x)$, then there is a member $M$ of $N S(x)$ such that $M \subseteq N$ and $M \in N S(y)$ for each $y \in M$ (that is, $M$ is a neighborhood of each of its points).

Omitting (TN 4) leads to a so-called pretopological neighborhood system on $U$.

\section{Total pure reflexive neighborhood systems}

A neighborhood system $N S: U \longrightarrow 2^{2^{U}}$ in which each $x \in U, N S(x)$ satisfying axiom (TN 1) will be referred to as a total pure reflexive (TPR) neighborhood system on $U$.

Definition $2([2,11])$. Let $N S: U \longrightarrow 2^{2^{U}}$ be a TPR neighborhood system. For each subset $X$ of $U$, the lower and upper approximations of $X, \underline{N S}(X)$ and $\overline{N S}(X)$, respectively, are defined as follows:

$$
\begin{aligned}
& \underline{N S}(X)=\{x \in U \mid N \subseteq X, \text { for some } N \in N S(x)\} \\
& \overline{N S}(X)=\{x \in U \mid N \cap X \neq \emptyset, \quad \forall N \in N S(x)\} .
\end{aligned}
$$

The lower and upper approximations are mutually dual in the sense that

$$
\overline{N S}(X)=U-\underline{N S}(U-X), \quad \forall X \subseteq U
$$

Lemma 2. Let $N S: U \longrightarrow 2^{2^{U}}$ be a TPR neighborhood system. Then:

1. $\overline{N S}(\emptyset)=\emptyset$.

2. $X \subseteq \overline{N S}(X), \forall X \subseteq U$. 
3. $X \subset Y \subseteq U$ implies $\overline{N S}(X) \subseteq \overline{N S}(Y)$ or equivalently:

$\overline{N S}(X \cup Y) \supseteq \overline{N S}(X) \cup \overline{N S}(Y)$ for all nonempty $X, Y \subseteq U$.

Proof. Item 1 follows immediately from (3.7).

To prove Item 2, considering Items 1, we just need to prove

$$
X \subseteq \overline{N S}(X), \quad \forall X \neq \emptyset
$$

Suppose that $X$ is a given nonempty subset of $U$ and $x \in X$. If follows from (3.7) and axiom (TN 1) that $x \in \overline{N S}(X)$. This gives (3.9).

Item 3 follows immediately from (3.7).

Given a TPR neighborhood system $N S: U \longrightarrow 2^{2^{U}}$, we consider a new neighborhood system $L N S: U \longrightarrow 2^{2^{U}}$, due to Lin [9], as follows: Let $x \in U$, if $M \supseteq N$ for a neighborhood $N \in N S(x)$, then $M \in L N S(x)$. In other words,

$$
L N S(x)=\{M \mid M \supseteq N \text { for some } N \in N S(x)\}
$$

The TPR neighborhood system $L N S: U \longrightarrow 2^{2^{U}}$ thus generated will be referred to as the pre-topologically maximal neighborhood system of $N S$ : $U \longrightarrow 2^{2^{U}}$.

Lemma 3. Let $N S: U \longrightarrow 2^{2^{U}}$ be a TPR neighborhood system, and let $L N S: U \longrightarrow 2^{2^{U}}$ be its pre-topologically maximal neighborhood system. Then

1. $\underline{L N S}(X)=\underline{N S}(X)$ and $\overline{L N S}(X)=\overline{N S}(X), \forall X \subseteq U$.

2. $\{X \subseteq U \mid \underline{N S}(X)=X\}=\{X \subseteq U \mid X \in L N S(x)$ whenever $x \in X\}$.

Proof. Item 1 follows immediately from (3.6), (3.7), and (3.10).

We show Item 2 as follows: It is clear that the empty set belong to both collections. Assume that $\underline{N S}(X)=X$ and $x \in X$, we have by (3.6) that there is an $N \in N S(x)$ such that $N \subseteq X$; by (3.10), $X \in L N S(x)$. This gives

$\{X \subseteq U \mid \underline{N S}(X)=X\} \subseteq\{X \subseteq U \mid X \in L N S(x)$ whenever $x \in X\}$.

We next show that

$\{X \subseteq U \mid \underline{N S}(X)=X\} \supseteq\{X \subseteq U \mid X \in L N S(x)$ whenever $x \in X\}$. 
Now let $X \in\{X \subseteq U \mid X \in L N S(x)$ whenever $x \in X\}$ and $X \neq \emptyset$. We have by Item 1 and (3.6) that $X \subseteq \underline{L N S}(X)=\underline{N S}(X)$ and therefore, using duality and the extensivity of the upper approximation $\overline{N S}: 2^{U} \rightarrow 2^{U}$, we obtain $\underline{N S}(X)=X$.

\section{Preclosures, preinteriors, and pretopological neighborhood systems}

By a preclosure on $U$ we mean an operator $c: 2^{U} \rightarrow 2^{U}$ fulfilling (KC 1 ), ( $\mathrm{KC} 2$ ), and ( $\mathrm{KC} 4$ ), but not necessarily ( $\mathrm{KC} 3$ ). That is, a preclosure is similar to a topological closure, except that it is not required to be idempotent.

\subsection{Topologies induced from preclosures}

Let $c: 2^{U} \rightarrow 2^{U}$ and $i: 2^{U} \rightarrow 2^{U}$ be a preclosure and its dual preinterior on $U$. A set $N \subseteq U$ is a neighborhood of $x \in U$ if and only if $x \in U-c(U-N)$. Let $N S(x)$ be the family of all neighborhoods of $x$. It can be shown that the family of all subsets $X$ of $U$ for which $c(U-X)=U-X$ is a topology on $U$, and that the induced neighborhood system $N S: x \longmapsto N S(x)$ is a pretopological neighborhood system on $U$. More precisely:

Lemma 4. Let $c: 2^{U} \rightarrow 2^{U}$ and $i: 2^{U} \rightarrow 2^{U}$ be a preclosure and its dual preinterior on $U$, and let

$$
\mathcal{F}_{c}=\{X \subseteq U \mid c(X)=X\}, \quad \mathcal{T}_{c}=\{(U-X) \subseteq U \mid c(X)=X\}
$$

Then

1. the family $\mathcal{T}_{c}$ of complements of members of $\mathcal{F}_{c}$ is a topology on $U$.

2. $\mathcal{T}_{c}=\{X \subseteq U \mid i(X)=X\}$.

3. the induced neighborhood system

$$
N S: x \longmapsto N S(x)=\{N \subseteq U \mid x \in i(N)\}, \quad x \in U
$$

is a pretopological neighborhood system on $U$.

If in addition $c: 2^{U} \rightarrow 2^{U}$ satisfies the axiom of idempotence, then

$$
\mathcal{F}_{c}=\{c(X) \mid X \subseteq U\}, \quad \mathcal{T}_{c}=\{i(X) \mid X \subseteq U\}
$$


Proof. The proof for Item 1 can be achieved using similar arguments given by Kelley [5] for Kuratowski closures.

By axiom (KC 1) the empty set belongs to $\mathcal{F}_{c}$; by axiom (KC 2) $U \subseteq$ $c(U)$; hence $U=c(U)$. This gives $U \in \mathcal{F}_{c}$. Axiom (KC 4) shows that $\mathcal{F}_{c}$ is closed under binary unions; consequently, $\mathcal{F}_{c}$ is closed under finite unions. It will follow that $\mathcal{T}_{c}$ is a topology for $U$ if it is shown that $\mathcal{F}_{c}$ is closed under arbitrary intersections.

By convention, $\bigcap_{\emptyset}=U$ and therefore, it suffices to show that the intersection of the members of any nonempty subfamily of $\mathcal{F}_{c}$ is a member of $\mathcal{F}_{c}$.

We now let $\mathcal{A}$ be a nonempty subfamily of $\mathcal{F}_{c}$, and let $B=\bigcap_{A \in \mathcal{A}} A$. We then have $B \subseteq A$ for all $A \in \mathcal{A}$, and therefore, from axiom (KC 5), $c(B) \subseteq c(A)$ for all $A \in \mathcal{A}$. This gives $c(B) \subseteq \bigcap_{A \in \mathcal{A}} c(A)=\bigcap_{A \in \mathcal{A}} A=B$, which implies that $c(B)=B$ and $B \in \mathcal{F}_{c}$. This shows that $\mathcal{T}_{c}$ is a topology.

Item 2 follows from the observation: $c(X)=X \Longleftrightarrow i(U-X)=$ $U-X, \forall X \subseteq U$.

We show Item 3 as follows.

(i) Let $x \in U$. If $N \in N S(x)$ then by (4.12), $x \in i(N)$; hence, by axiom (KI 2), we have $x \in N$.

(ii) If $N, M$ are members of $N S(x)$ then by (4.12), $x \in i(N)$ and $x \in$ $i(M)$. Hence $x \in i(N) \cap i(M)$; by (KI 4$), x \in i(N \cap M)$. This gives $N \cap M \in$ $N S(x)$.

(iii) We have seen that (KI 4) implies (KI 5). Let $x \in U$. If $M \supseteq N$ for a nonempty $N \in N S(x)$ then by (4.12), $x \in i(N)$. By (KI 5$), x \in i(M)$. This gives $M \in N S(x)$.

If in addition $c: 2^{U} \rightarrow 2^{U}$ is idempotent, then (4.13) follows immediately from (4.11), Item 1 and Item 2.

\subsection{Topologies from pretopological neighborhood systems}

Let $N S: U \longrightarrow 2^{2^{U}}$ be a pretopological neighborhood system. We show that the associated upper approximation $\overline{N S}: 2^{U} \longrightarrow 2^{U}$ is a preclosure on $U$. By Lemma 2, it suffices to show that

$$
\overline{N S}(X \cup Y) \subseteq \overline{N S}(X) \cup \overline{N S}(Y), \quad \forall X, Y \subseteq U .
$$

By contradiction, suppose that $x \notin \overline{N S}(X) \cup \overline{N S}(Y)$. Then

$$
x \in U-(\overline{N S}(X) \cup \overline{N S}(Y))=(U-\overline{N S}(X)) \cap(U-\overline{N S}(Y)) .
$$


By (3.7), there exist $N_{1}, N_{2} \in N S(x)$ such that $N_{1} \cap X=\emptyset$ and $N_{2} \cap Y=\emptyset$; consequently, $N_{1} \subseteq(U-X)$ and $N_{2} \subseteq(U-Y)$. Thus $\left(N_{1} \cap N_{2}\right) \subseteq(U-$ $X) \cap(U-Y)=U-(X \cup Y)$; therefore $\left(N_{1} \cap N_{2}\right) \cap(X \cup Y)=\emptyset$.

Also, by axioms (TN 1) and (TN 2), $x \in\left(N_{1} \cap N_{2}\right) \in N S(x)$; hence $x \notin \overline{N S}(X \cup Y) . \overline{N S}: 2^{U} \rightarrow 2^{U}$ is therefore a preclosure on $U$. This fact, combined with Lemmas 3 and 4 , leads to the following:

Theorem 1. Let $N S: U \longrightarrow 2^{2^{U}}$ be a pretopological neighborhood system. Then

1. the induced upper approximation $\overline{N S}: 2^{U} \rightarrow 2^{U}$ is a preclosure on $U$.

2. the family $\mathcal{T}$ of all subsets $X$ of $U$ for which $\underline{N S}(X)=X$ forms a topology on $U$.

3. a subset $X$ of $U$ belongs to $\mathcal{T}$ if and only if it is a neighborhood of each of its points.

We have thus seen from Lemma 4 that preclosures, preinteriors, and pretopological neighborhood systems are equivalent constructions on the universe $U$.

\section{Approximation in reflexive relations}

Unless otherwise specified, we assume in the following that $R \subseteq U \times U$ is reflexive. In rough set community, $(2.5)$ is directly generalized to a reflexive relation $R$ by interpreting $[x]_{\beta}$ as $R(x)$ [15].

Definition 3 ([15]). Let $R \subseteq U \times U$ be reflexive. For any $X \subseteq U$, the lower and upper approximations, $R_{*}(X)$ and $R^{*}(X)$, are respectively defined by

$$
R_{*}(X)=\{x \in U \mid R(x) \subseteq X\}, \quad R^{*}(X)=\{x \in U \mid R(x) \cap X \neq \emptyset\}
$$

Observe from (5.14) that the reflexive relation $R$ can be regarded as the TPR neighborhood system $x \longmapsto\{R(x)\}$. Using duality and Lemma 2 , we obtain the following:

Lemma 5. Considering a reflexive relation $R$ on $U$, we have:

1. $R_{*}(X)=U-R^{*}(U-X), \forall X \subseteq U$.

2. $R^{*}(\emptyset)=\emptyset ; R_{*}(U)=U$.

3. $R_{*}(X) \subseteq X \subseteq R^{*}(X), \forall X \subseteq U$.

4. If $X \subseteq Y \subseteq U$, then $R_{*}(X) \subseteq R_{*}(Y)$ and $R^{*}(X) \subseteq R^{*}(Y)$. 


\subsection{Alexandroff topologies induced by reflexive relations}

Considering a reflexive relation $R$ on $U$, it is useful to observe from (5.14) that for any nonempty subfamily $\mathcal{A}$ of $2^{U}, R^{*}\left(\bigcup_{X \in \mathcal{A}} X\right)=\bigcup_{X \in \mathcal{A}} R^{*}(X)$. By convention, $\bigcup_{\emptyset}=\emptyset$, it follows that

$$
R^{*}\left(\bigcup_{X \in \mathcal{A}} X\right)=\bigcup_{X \in \mathcal{A}} R^{*}(X), \quad \forall \mathcal{A} \subseteq 2^{U}
$$

Using duality and (5.15), we obtain

$$
R_{*}\left(\bigcap_{X \in \mathcal{A}} X\right)=\bigcap_{X \in \mathcal{A}} R_{*}(X), \quad \forall \mathcal{A} \subseteq 2^{U} .
$$

It follows from Lemma 5 and (5.15) that the upper approximation $R^{*}$ : $2^{U} \longrightarrow 2^{U}$ associated with the reflexive relation $R$ is a preclosure on $U$. According to Lemma 4 , the collection $\mathcal{T}_{R}=\left\{X \subseteq U \mid R_{*}(X)=X\right\}$ is a topology on $U$.

We next show that $\mathcal{T}_{R}$ is an Alexandroff topology by showing that the intersection of the members of any nonempty subfamily of $\mathcal{T}_{R}$ is a member of $\mathcal{T}_{R}$. Let $\mathcal{A}$ be a nonempty subfamily of $\mathcal{T}_{R}$, and let $B=\bigcap_{A \in \mathcal{A}} A$. By (5.16), we obtain

$$
R_{*}(B)=R_{*}\left(\bigcap_{A \in \mathcal{A}} A\right)=\bigcap_{A \in \mathcal{A}} R_{*}(A)=\bigcap_{A \in \mathcal{A}} A=B \text {; hence } B \in \mathcal{T}_{R} .
$$

According to Speer [16], the minimal open neighborhoods are the natural objects of study in an Alexandroff space. In the Alexandroff space $\left(U, \mathcal{T}_{R}\right)$, we might naturally ask, "For each $x \in U$, is $R(x)$, or what is, the smallest open neighborhood of $x$ ?"

We have seen that preclosures and pretopological neighborhood systems are equivalent constructions on the universe $U$.

According to (3.10), the pretopological neighborhood system

$$
x \longmapsto N S(x)=\{N \subseteq U \mid N \supseteq R(x)\}
$$

is the pre-topologically maximal neighborhood system of $x \longmapsto\{R(x)\}$. Based on the associated pretopological neighborhood system, it is clear that the smallest $\mathcal{T}_{R}$-open neighborhood of each object $x$ is the intersection of all $\mathcal{T}_{R^{-}}$open sets containing $R(x)$.

We summarize the results of this discussion in the following theorem. 
Theorem 2. Considering a reflexive relation $R$ on $U$, let

$$
\mathcal{T}_{R}=\left\{X \subseteq U \mid R_{*}(X)=X\right\}
$$

Then

1. the upper approximation $R^{*}: 2^{U} \rightarrow 2^{U}$ is a preclosure on $U$.

2. the collection $\mathcal{T}_{R}=\left\{X \subseteq U \mid R_{*}(X)=X\right\}$ is an Alexandroff topology on $U$.

3. the induced pretopological neighborhood system of $R^{*}: 2^{U} \rightarrow 2^{U}$ is given by

$$
x \longmapsto N S(x)=\{N \subseteq U \mid N \supseteq R(x)\}, \quad \forall x \in U .
$$

That is, NS:U $\longrightarrow 2^{2^{U}}$ defined by (5.18) is a pretopological neighborhood system and

$$
\underline{N S}(X)=R_{*}(X) \text { and } \overline{N S}(X)=R^{*}(X), \quad \forall X \subseteq U .
$$

4. for each $x \in U$, let $S(x)$ be the smallest open neighborhood of $x$ in the Alexandroff space $\left(U, \mathcal{T}_{R}\right)$. Then

$$
S(x)=\cap\left\{N \subseteq U \mid N \supseteq R(x) \text { and } R_{*}(N)=N\right\} .
$$

Remark. It is proved in Theorem 3.1 of [18] that the family

$$
T=\left\{X \subseteq U \mid R_{*}(X)=R^{*}(X)\right\}
$$

is a topology on $U$. Let $\mathcal{F}_{R}=\left\{X \subseteq U \mid R^{*}(X)=X\right\}$. Then, from (5.17) and (5.21), we obtain $T=\mathcal{T}_{R} \cap \mathcal{F}_{R}$.

Considering a reflexive and transitive relation $R \subseteq U \times U$ and an object $x \in U$, we have $R(x) \neq \emptyset$ and

$$
R(y) \subseteq R(x), \quad \forall y \in R(x) .
$$

It is thus seen that $R(x)$ is $\mathcal{T}_{R}$-open; consequently, the neighborhood system

$$
x \longmapsto N S(x)=\{N \subseteq U \mid N \supseteq R(x)\}, \quad \forall x \in U .
$$

is a topological neighborhood system on $U$. According to (4.13) and Theorem 2, we have an immediate consequence as follows.

Corollary 1. Considering a reflexive and transitive relation $R$ on $U$, we have 
1. the upper approximation $R^{*}: 2^{U} \rightarrow 2^{U}$ is a closure on $U$.

2. the collection $\mathcal{T}_{R}=\left\{X \subseteq U \mid R_{*}(X)=X\right\}$ is an Alexandroff topology on $U$.

3. for each $x \in U, R(x)$ is its smallest $\mathcal{T}_{R}$-open neighborhood.

4. the induced neighborhood system of $R^{*}: 2^{U} \rightarrow 2^{U}$ given by

$$
x \longmapsto N S(x)=\{N \subseteq U \mid N \supseteq R(x)\}
$$

is a topological neighborhood system on $U$.

5. $\mathcal{T}_{R}=\left\{R_{*}(X) \mid X \subseteq U\right\}$.

Example 1. Consider a set $U=\left\{x_{1}, x_{2}, x_{3}, x_{4}\right\}$ and a reflexive relation

$$
R=\left\{\left(x_{1}, x_{1}\right),\left(x_{2}, x_{2}\right),\left(x_{3}, x_{3}\right),\left(x_{4}, x_{4}\right),\left(x_{1}, x_{2}\right),\left(x_{3}, x_{4}\right),\left(x_{4}, x_{2}\right)\right\} .
$$

$R$ is not symmetric or transitive. The images are:

$R\left(x_{1}\right)=\left\{x_{1}, x_{2}\right\}, R\left(x_{2}\right)=\left\{x_{2}\right\}, \quad R\left(x_{3}\right)=\left\{x_{3}, x_{4}\right\}$ and $R\left(x_{4}\right)=\left\{x_{2}, x_{4}\right\}$.

From these images, we derive the lower and upper approximations as shown in Table 1.

From Table 1, we have

$\mathcal{T}_{R}=\left\{\emptyset,\left\{x_{2}\right\},\left\{x_{1}, x_{2}\right\},\left\{x_{2}, x_{4}\right\},\left\{x_{1}, x_{2}, x_{4}\right\},\left\{x_{2}, x_{3}, x_{4}\right\},\left\{x_{1}, x_{2}, x_{3}, x_{4}\right\}\right\}$,

which can be easily checked to be an Alexandroff topology. From (5.22) and Table 1, we observe that:

- the images $R\left(x_{1}\right), R\left(x_{2}\right)$ and $R\left(x_{4}\right)$ are open.

- the image $R\left(x_{3}\right)$ is closed but not open.

- the smallest open neighborhoods are:

$$
\begin{aligned}
& S\left(x_{1}\right)=\left\{x_{1}, x_{2}\right\}, \quad S\left(x_{2}\right)=\left\{x_{2}\right\}, \\
& S\left(x_{3}\right)=\left\{x_{2}, x_{3}, x_{4}\right\} \text { and } S\left(x_{4}\right)=\left\{x_{2}, x_{4}\right\} .
\end{aligned}
$$

\subsection{Definability}

It is proved in Result 12 of [15] that for a binary relation $R$ on a finite universe, an $R$-definable set $X$ has a unique reference set of maximal cardinality. We observe that the arguments in proving this result are still valid for infinite universe. This observation leads to the definition of maximal reference sets of $R$-definable sets as follows: 
Table 1: The lower and Upper Approximations

\begin{tabular}{|c|c|c|}
\hline$X$ & $R_{*}(X)$ & $R^{*}(X)$ \\
\hline \hline$\emptyset$ & $\emptyset$ & $\emptyset$ \\
\hline$\left\{x_{1}\right\}$ & $\emptyset$ & $\left\{x_{1}\right\}$ \\
\hline$\left\{x_{2}\right\}$ & $\left\{x_{2}\right\}$ & $\left\{x_{1}, x_{2}, x_{4}\right\}$ \\
\hline$\left\{x_{3}\right\}$ & $\emptyset$ & $\left\{x_{3}\right\}$ \\
\hline$\left\{x_{4}\right\}$ & $\emptyset$ & $\left\{x_{3}, x_{4}\right\}$ \\
\hline$\left\{x_{1}, x_{2}\right\}$ & $\left\{x_{1}, x_{2}\right\}$ & $\left\{x_{1}, x_{2}, x_{4}\right\}$ \\
\hline$\left\{x_{1}, x_{3}\right\}$ & $\emptyset$ & $\left\{x_{1}, x_{3}\right\}$ \\
\hline$\left\{x_{1}, x_{4}\right\}$ & $\emptyset$ & $\left\{x_{1}, x_{3}, x_{4}\right\}$ \\
\hline$\left\{x_{2}, x_{3}\right\}$ & $\left\{x_{2}\right\}$ & $\left\{x_{1}, x_{2}, x_{3}, x_{4}\right\}$ \\
\hline$\left\{x_{2}, x_{4}\right\}$ & $\left\{x_{2}, x_{4}\right\}$ & $\left\{x_{1}, x_{2}, x_{3}, x_{4}\right\}$ \\
\hline$\left\{x_{3}, x_{4}\right\}$ & $\left\{x_{3}\right\}$ & $\left\{x_{3}, x_{4}\right\}$ \\
\hline$\left\{x_{1}, x_{2}, x_{3}\right\}$ & $\left\{x_{1}, x_{2}\right\}$ & $\left\{x_{1}, x_{2}, x_{3}, x_{4}\right\}$ \\
\hline$\left\{x_{1}, x_{2}, x_{4}\right\}$ & $\left\{x_{1}, x_{2}, x_{4}\right\}$ & $\left\{x_{1}, x_{2}, x_{3}, x_{4}\right\}$ \\
\hline$\left\{x_{1}, x_{3}, x_{4}\right\}$ & $\left\{x_{3}\right\}$ & $\left\{x_{1}, x_{3}, x_{4}\right\}$ \\
\hline$\left\{x_{2}, x_{3}, x_{4}\right\}$ & $\left\{x_{2}, x_{3}, x_{4}\right\}$ & $\left\{x_{1}, x_{2}, x_{3}, x_{4}\right\}$ \\
\hline$\left\{x_{1}, x_{2}, x_{3}, x_{4}\right\}$ & $\left\{x_{1}, x_{2}, x_{3}, x_{4}\right\}$ & $\left\{x_{1}, x_{2}, x_{3}, x_{4}\right\}$ \\
\hline
\end{tabular}

Definition 4. Considering a binary relation $R$ on $U$ and an $R$-definable set $X$ of $U$. A reference set $X^{\prime \prime}$ of $X$ is called the maximal reference set of $X$ if it contains any reference set of $X$. That is, $X^{\prime \prime} \supseteq X^{\prime}$ for any reference set $X^{\prime}$ of $X$.

Considering a reflexive relation $R$ on $U$ and a subset $X$ of $U$, the relation

$$
\left\{x \in U \mid R(x) \subseteq X \text { and } R^{-1}(x) \subseteq X\right\}=R_{*}(X) \cap\left(R^{-1}\right)_{*}(X)
$$

follows from

$$
\begin{aligned}
x & \in\left\{x \in U \mid R(x) \subseteq X \text { and } R^{-1}(x) \subseteq X\right\} \\
& \Longleftrightarrow \quad R(x) \subseteq X \text { and } R^{-1}(x) \subseteq X \\
& \Longleftrightarrow x \in R_{*}(X) \text { and } x \in\left(R^{-1}\right)_{*}(X) \\
& \Longleftrightarrow x \in R_{*}(X) \cap\left(R^{-1}\right)_{*}(X) .
\end{aligned}
$$

According to (5.14), together with Lemma 1 and Result 12 in [15], we obtain the following:

Theorem 3. Considering a reflexive relation $R$ on $U$ and a subset $X$ of $U$, let

$$
X^{\prime \prime}=R_{*}(X) \cap\left(R^{-1}\right)_{*}(X) .
$$


Then

1. $X$ is $R$-definable if and only if $R^{*}\left(X^{\prime \prime}\right)=X$.

2. If $X$ is $R$-definable, then $X^{\prime \prime}$ defined by (5.24) is the maximal reference set of $X$.

Given a reflexive relation $R \subseteq U \times U$, we associate it with the equivalence relation $E$ induced by its associated neighborhood system $x \longmapsto\{R(x)\}$. That is, $(x, y) \in E$ if and only if $R(x)=R(y)$. Accordingly, for any $X \subseteq U$, $R_{*}(X)$ and $R^{*}(X)$ can be also described as shown below:

$$
\begin{gathered}
R_{*}(X)=\cup\left\{[x]_{E} \in U / E \mid R(x) \subseteq X\right\}, \\
R^{*}(X)=\cup\left\{[x]_{E} \in U / E \mid R(x) \cap X \neq \emptyset\right\} .
\end{gathered}
$$

According to Theorem 3, we have immediate consequences as follows.

Corollary 2. Considering a reflexive and symmetric relation $R$ on $U$ and a subset $X$ of $U$, then $X$ is $R$-definable if and only if $R^{*}\left(R_{*}(X)\right)=X$.

Corollary 3. Considering a reflexive relation $R$ on $U$ and a subset $X$ of $U$, if $X$ is $R$-definable then it can be expressed as a union of members of $U / E$.

Example 2. Consider a set $U=\left\{x_{1}, x_{2}, x_{3}, x_{4}\right\}$ and a reflexive relation

$$
R=\left\{\left(x_{1}, x_{1}\right),\left(x_{2}, x_{2}\right),\left(x_{3}, x_{3}\right),\left(x_{4}, x_{4}\right),\left(x_{1}, x_{2}\right),\left(x_{2}, x_{1}\right),\left(x_{3}, x_{4}\right),\left(x_{4}, x_{2}\right)\right\} .
$$

$R$ is not symmetric or transitive. The images of $R$ and $R^{-1}$ are:

$$
\begin{array}{ll}
R\left(x_{1}\right)=\left\{x_{1}, x_{2}\right\}, & R^{-1}\left(x_{1}\right)=\left\{x_{1}, x_{2}\right\} \\
R\left(x_{2}\right)=\left\{x_{1}, x_{2}\right\}, & R^{-1}\left(x_{2}\right)=\left\{x_{1}, x_{2}, x_{4}\right\} \\
R\left(x_{3}\right)=\left\{x_{3}, x_{4}\right\}, & R^{-1}\left(x_{3}\right)=\left\{x_{3}\right\} \\
R\left(x_{4}\right)=\left\{x_{2}, x_{4}\right\}, & R^{-1}\left(x_{4}\right)=\left\{x_{3}, x_{4}\right\} .
\end{array}
$$

From the images of $R$, we have

$$
X / E=\left\{\left\{x_{1}, x_{2}\right\},\left\{x_{3}\right\},\left\{x_{4}\right\}\right\} .
$$

In this example, the set $X=\left\{x_{2}, x_{3}, x_{4}\right\}$ is not $R$-definable.

- This can been using Item 1 of Theorem 3. We indeed have $X^{\prime \prime}=$ $\left\{x_{3}, x_{4}\right\}$ and $R^{*}\left(\left\{x_{3}, x_{4}\right\}\right) \neq\left\{x_{2}, x_{3}, x_{4}\right\}$.

- This can be easily checked using Corollary 3 . Indeed, the set $X=$ $\left\{x_{2}, x_{3}, x_{4}\right\}$ cannot be expressed as a union of members of $U / E$.

Consider now $X=\left\{x_{1}, x_{2}, x_{4}\right\}$. Here $X$ is $R$-definable since $X^{\prime \prime}=$ $\left\{x_{1}, x_{2}\right\}$ and $R^{*}\left(\left\{x_{1}, x_{2}\right\}\right)=X$. 


\section{Conclusions}

Considering a reflexive relation $R$ on a fixed nonempty set $U$, we use a special kind of neighborhood systems, called total pure reflexive neighborhood systems, to investigate the structure of the approximation space $(U, R)$. We show that the family of all subsets $X$ of $U$ for which $R^{*}(U-X)=U-X$ is an Alexandroff topology for $U$, and that the pre-topologically maximal neighborhood system of the neighborhood system $x \longmapsto\{R(x)\}$ associated to $R$ is exactly the pretopological neighborhood system whose induced upper approximation is identical to $R^{*}$. Accordingly, we show that for each $x \in U$, its smallest open neighborhood is the intersection of all open sets containing $R(x)$. We also study the notion of $R$-definability [15] in rough approximation theory. We establish a characterization of definable sets in terms of $R^{*}$ and its dual $R_{*}$. This result would help explore the concept of "definability" in neighborhood system approximation theory. In addition, we give a necessary condition for $R$-definability.

Approximation spaces $(U, R)$ with the property of $R$ being reflexive, and reflexive and symmetric, have applications to incomplete information systems [14]. i.e. to systems in which attribute values for objects may be unknown (missing, null). In [7], Kryszkiewicz introduced the use of a symmetric similarity relation to deal with the missing value case. In [17], Stefanowski and Tsoukias introduced the use of a non symmetric similarity (reflexive) relation to formalize the idea of absent value semantics. It is our intent to obtain similar applications under $R$-definability condition.

\section{References}

[1] F. G. Arenas (1999) Alexandroff spaces, Acta Mathematica Universitatis Comenianae, LXVIII, 17-25. MR1711071

[2] G. Cattaneo (1998) Abstract approximation spaces for rough theories, in: L. Polkowski and A. Skowron (Eds.) Rough Sets in Knowledge Discovery 1: Methodology and Applications, Studies in Fuzziness and Soft Computing, vol. 18 (pp. 59-98) Physica-Verlag, Heidelberg. MR1679370

[3] M. M. Day (1944) Convergence, closure, and neighborhoods, Duke Mathematical Journal, 11, 181-199. MR0009844

[4] J. W. Grzymala-Busse, W. Rzasa (2010) Definability and other properties of approximations for generalized indiscernibility relations, in: J. F. Peters and A. Skowron (Eds.) Transactions on Rough Sets XI (pp. 14-39) Springer-Verlag. 
[5] J. L. Kelley (1955) General topology. Van Nostrand, (Chapter 1). MR0070144

[6] M. Kondo (2005) On the structure of generalized rough sets, Information Sciences, 176 (5), 589-600. MR2200461

[7] M. Kryszkiewicz (1998) Rough set approach to incomplete information system, Information Sciences, 112, 39-49. MR1655891

[8] T. Y. Lin (1998) Granular computing on binary relations I: data mining and neighborhood systems, in: A. Skowron and L. Polkowski (Eds.) Rough sets and knowledge discovery (pp. 107-121) Physica-Verlag. MR1679372

[9] T. Y. Lin (1992) Topological and fuzzy rough sets, in: R. Slowinski (Ed.) Intelligent Decision Support - Handbook of Applications and Advances of the Rough Sets theory (pp. 287-304) Kluwer Academic, Dordrecht.

[10] T. Y. Lin, Y. R. Syau, Keynote Speech: Granular mathematics - foundation and current state, in: Proceedings of the 2011 IEEE International Conference on Granular Computing, 2011, pp. 4-12.

[11] T. Y. Lin, Y. R. Syau (2013) Unifying variable precision and classical rough sets: granular approach, in: A. Skowron and Z. Suraj (Eds.) Rough Sets and Intelligent Systems - Professor Zdzislaw Pawlak in Memoriam (pp. 287-304) In series: Intelligent Systems Reference Library, vol. 43, Springer, Berlin/Heidelberg.

[12] Z. Pawlak (1991) Rough sets: theoretical aspects of reasoning about data. Kluwer Academic Publishers, Dordrecht, (Chapters 1 \& 2).

[13] Z. Pawlak (1982) Rough sets, International Journal of Computer and Information Science, 11, 341-356. MR0703291

[14] R. Slowinski, J. Stefanowski (1989) Rough classification in incomplete information systems, Mathematical and Computer Modelling, 12 (10/11), 1347-1357.

[15] R. Slowinski, D. Vanderpooten (2000) A Generalized definition of rough approximations based on similarity, IEEE Transactions on Data and Knowledge Engineering, 12, 331-336.

[16] T. Speer (2007) A Short Study of Alexandroff Spaces, arXiv: 0708.2136v1, New York University.

[17] J. Stefanowski, A. Tsoukias (2001). Incomplete information tables and rough classification, Computational Intelligence, 17, 545-566. 
[18] H. Yu, W. R. Zhan (2013) On the topological properties of generalized rough sets, to appear in Information Sciences. MR3158690

[19] H. P. Zhang, Y. Ouyang, Z. Wang (2009) Note on "Generalized rough sets based on reflexive and transitive relations", Information Sciences, 179, 471-473. MR2493788

Yu-Ru SYAU

Department of Information MANAGEMENT

NATIONAL Formosa University

HuWEi 63201, YunLin

TAIWAN

LIXING JIA

Department of Mathematics \& Computer Science

Chicago State University

ChicAgo, IL 60628

USA

E-mail address: ljia@csu.edu

Received August 25, 2013 